\title{
Schweigen über Differenz. Schwarze im kolonialen und postkolonialen Sicherheitsdiskurs Brasiliens
}

\author{
Jorun Poettering
}

\begin{abstract}
In 1808, Portugal's royal court fled from Napoleon's forces and resettled in Rio de Janeiro. Within a very short time, the city's population grew rapidly while it also had to meet the demands of a European royal capital. This situation fostered two different security discourses. On the one hand, there was a discourse, also widespread throughout much of Europe, on the threat posed by mostly foreign revolutionaries. But, as this contribution shows, on the other hand, there was a telling silence about potentially insurgent black people. This silence was only broken once they became the target of repression by the public security apparatus and more and more frequently struggled to establish their rights (and thus their security). However, the abolition of slavery in 1888 intensified racism, which once again became covered by public silence - a silence that willfully ignored the difference constituted by this very racism and thus continued to obstruct security for the black population.
\end{abstract}

"Security makes a difference“ - Sicherheitsdiskurse und Differenzdiskurse bedingen einander, so Sigrid Ruby in ihrer Einleitung. ${ }^{1}$ Mit Lene Hansen und Sarah Bertrand folgert Ruby weiter: Subalterne Menschen befinden sich oft in einem Sicherheitsdilemma, denn sie können nicht über ihre Unsicherheit sprechen, ohne ihre Differenz zu bestätigen und sich noch weiter zu gefährden (Hansen 2000; Bertrand 2018; vgl. auch Spivak 1988/2016). Am Beispiel der brasilianischen Sklaverei des 19. Jahrhunderts und der Differenz zwischen Versklavten/Schwarzen und Freien/Weißen möchte ich zeigen, dass das Schweigen über Differenz keine sinnvolle Alternative ist, da es nicht zur Auflösung der differenzbasierten Unsicherheit, sondern vielmehr zu ihrer Beibehaltung, wenn nicht Konsolidierung beiträgt. Das Sprechen über Differenz, in diesem Fall über die Existenz und die Seinsbedingungen zunächst von versklavten, dann aber auch von

1 Dieser Aufsatz ist aus einem Forschungsprojekt erwachsen, das unter anderem von der Gerda Henkel Stiftung, dem EU-Förderprogramm für Forschung und Innovation Horizon 2020 (Marie Skłodowska-Curie Zuwendungsvereinbarung Nr. 659520) und der Alexander von Humboldt Stiftung gefördert wurde. 
freien schwarzen Menschen, kann dagegen ermächtigend und integrierend wirken, die gesellschaftliche Differenz verringern und Sicherheit erzeugen. An der Abschaffung der Sklaverei in Brasilien lässt sich beobachten, dass subalterne Gruppen Sicherheit einklagen können, wenn es ihnen gelingt, das Schweigen über Differenz zu brechen. Allerdings handelt es sich dabei oft um langwierige und von Rückschlägen begleitete Prozesse. Hegemoniale Gruppen reagieren darauf mit dem Setzen neuer Formen von Differenz - in meinem Beispiel mit der Überlagerung und schließlich Ersetzung der Sklaverei durch Rassismus. Diese neuen Formen von Differenz werden ebenfalls beschwiegen und tragen dadurch zur erneuten Entstehung von Unsicherheit für die Subalternen bei.

\section{Der brasilianische Sicherheitsdiskurs zu Beginn des 19. Jabrhunderts}

Ende des 18. Jahrhunderts fanden fast zeitgleich zwei Revolutionen von welthistorischer Bedeutung statt: die Französische Revolution und die Haitianische Revolution. Da die brasilianische Gesellschaft nicht nur von einem stark hierarchischen Ordnungsmodell bestimmt war, sondern zudem auf Sklaverei beruhte, sah ihre Elite in beiden Revolutionen eine potentielle Bedrohung auch für ihren eigenen Status. Doch das daraus abgeleitete Sicherheitsproblem formulierten ihre Mitglieder sowohl auf der diskursiven Ebene als auch bezüglich ihres praktischen Handelns vollkommen unterschiedlich.

Brasilien war zu diesem Zeitpunkt eine portugiesische Kolonie, seine Elite war eng mit der des Mutterlandes verbunden. Die Ratgeber der Krone beschäftigten sich früh mit der Gefahr, dass sich das umstürzlerische Gedankengut der Französischen Revolution auf das portugiesische Königreich und seine Überseegebiete ausbreiten könnte, und ergriffen spätestens mit der Übersiedlung des portugiesischen Hofes nach Rio de Janeiro im Jahr 1808 - auf der Flucht vor den napoleonischen Truppen - auch in Brasilien konkrete Maßnahmen zur Sicherung der etablierten Ordnung. Bereits einen Monat nach der Ankunft der königlichen Familie in der Neuen Welt gründete der Thronfolger João VI. eine Polizeiintendanz nach Lissabonner Vorbild, welche wiederum auf eine entsprechende Institution in Paris zurückging. Wenig später setzte er den Juristen Paulo Fernandes Viana als Generalintendanten ein und verlieh ihm umfangreiche Kompetenzen, um die "öffentliche Ruhe“ in der Stadt zu bewahren (Collecção 1891, S. 26f.). Laut den Statuten zählte dazu vor allem das Vorgehen gegen Bettelei und Vagabundentum sowie die Pflege der städtischen Infrastruktur, doch in der öffentlichen Wahrnehmung wurde das Amt insbesondere mit 
der Herstellung von Sicherheit durch die gezielte Überwachung von Ausländern identifiziert. Denn die Ereignisse in Frankreich wurden als grenzüberschreitende Bedrohung verstanden, die sich in Form von Ideen, Presse, Propaganda, Agenten, Geheimgesellschaften und Verschwörungen verbreiten und auch in Brasilien leicht in eine Revolution münden könnte (Härter 2013, S. 99). So betonte etwa der zeitgenössische Chronist Luís Gonçalves dos Santos (Padre Perereca), dass der Polizeiintendant von Rio „ein aufmerksamer Wächter über die öffentliche Sicherheit“ sei,

„der Spione und Parteigänger der Franzosen aus unseren Häusern vertreibt; auch wacht er über die geheimen Verbrechen, die im Dunkeln in Clubs und Geschäften ausgeheckt werden, bevor sie mit einer schrecklichen Explosion das offensichtliche Verderben des Staates, der Religion und sogar der Urheber der Explosion bewirken“. ${ }^{2}$

Zur Abwehr der „jakobinischen Gefahr“ organisierte der Polizeiintendant eine „feinmaschige Überwachung" (fina vigilancia), die sich nicht nur gegen Franzosen, sondern auch gegen andere Ausländer richtete, etwa gegen Spanier, die verdächtigt wurden, mit den Unabhängigkeitsbestrebungen und dem republikanischen Gedankengut in den spanischen Kolonien in Verbindung zu stehen (zit. n. Gerstenberger 2013, S. 163). In der Folge kam es immer wieder zu Verhaftungen von Personen, die der Auskundschaftung und Unterwanderung bezichtigt wurden. Die Historikerin Debora Gerstenberger spricht gar von „einem Netzwerk von Polizeispionen, die im Dienst der Regierung Ausländer und andere Verdächtige beschatteten" (ebd., S. 161).

Ein etwas weniger spektakuläres Mittel, welches die Polizeiintendanz zur Überwachung potentieller Aufrührer anwendete, war die Registrierung aller Reisenden, die im Hafen von Rio eintrafen oder diesen verließen. Sie wurden mit Einreisedatum, Name, Geburtsort, Personenbeschreibung, Tätigkeit, Angaben zu einer lokalen Kontaktperson und ähnlichen Informationen verzeichnet (Auler 1960). Für Reisen innerhalb des Landes (und ins Ausland) mussten sie einen Pass beantragen, so dass die Behörden den Aufenthaltsort der Ausländer theoretisch immer nachverfolgen konnten.

2, „[...] o qual fôsse um vigilante sentinela da segurança pública, fazendo arredar dos nossos lares os espiões, e partidaristas dos franceses; e também velasse sôbre os crimes secretos, que, forjados nas trevas em clubes, e lojas, arrebentam com explosão terrível com manifesta ruína do Estado, e da Religião, ou dos mesmos autores da explosão"; Santos 1943, S. 251. Meine Übersetzung. 
Anders als Gerstenbergers Darstellung suggeriert, handelt es sich bei dieser "Souveränitätspanik" nicht um ein neues Phänomen (Gerstenberger 2013, S. 172). Bis 1808 war allen Ausländern der Aufenthalt in der portugiesischen Kolonie grundsätzlich verboten gewesen, was nicht zuletzt Alexander von Humboldt daran gehindert hatte, das Land zu erkunden. Allein zu Versorgungszwecken hatten ausländische Schiffe in Rio einen Zwischenstopp einlegen dürfen; in zahlreichen Reiseberichten beschweren sich die wenigen Besatzungsmitglieder, die in solchen Fällen an Land gehen durften, über die kontinuierliche Überwachung, der sie dort ausgesetzt waren (Domingues 2013). Nach der Öffnung der Häfen für die befreundeten Nationen, die im Rahmen der Übersiedlung des portugiesischen Hofes erfolgte, wurde die Überwachung auf einer umfassenderen Ebene fortgesetzt. Dabei wandten die Behörden zumindest für das Stadtgebiet von Rio Techniken der Registrierung an, die mit den zeitgenössischen Techniken in Frankreich vergleichbar waren - dem Staat, dem John Torpey (2018) und andere Soziologen eine Vorreiterrolle bei der Einführung von Ausweisdokumenten und Grenzkontrollen zuschreiben. Die Vorstellung, dass die innere Sicherheit durch die Fokussierung auf die Überwachung der Landesgrenzen gewährleistet werden könne, ist ein Signum der zu dieser Zeit entstehenden Nationalstaaten; entsprechende Maßnahmen wurden auch an Orten der europäischen Peripherie wie Rio angewendet allerdings nur in Bezug auf die weiße Bevölkerung.

Denn eine Überwachung der schwarzen Bevölkerung gehörte nicht $\mathrm{zu}$ den Aufgaben, mit denen die Polizeioffiziere bei ihrer Einsetzung betraut worden (Collecção 1891, S. 11-16) oder die in der Wahrnehmung der Zeitgenossen besonders hervorgetreten wären - und dies trotz der Erfahrung der Haitianischen Revolution, die die Auswirkungen der Französischen Revolution in Bezug auf das Ausmaß an Gewalt und das politische Resultat noch deutlich übertraf. Versklavte und freie Schwarze der ehemaligen französischen Kolonie Saint Domingue hatten unzählige Sklavenhalter umgebracht, Plantagen niedergebrannt und die einst ertragreichste Kolonie der Karibik verwüstet. Nachdem die Auseinandersetzungen zunächst zur Abschaffung der Sklaverei geführt hatten, rief Haiti 1804 als erstes lateinamerikanisches Land seine politische Unabhängigkeit aus. Ehemals versklavte Personen hatten die herrschende Ordnung gestürzt und selbst die Macht ergriffen. Die schwarze Bevölkerung Brasiliens, dessen Wirtschafts- und Bevölkerungsstrukturen mit denen in Saint Domingue durchaus vergleichbar waren, hätte sich leicht von diesen Ereignissen inspirieren lassen können.

Den portugiesischen und brasilianischen Eliten waren die Ereignisse in Haiti nicht entgangen. Aber die Art, wie sie (ebenso wie die meisten 
anderen Menschen der bessergestellten sozialen Schichten in Europa und den Amerikas) über sie sprachen beziehungsweise über sie schwiegen, unterschied sich grundlegend von der diskursiven Behandlung der Französischen Revolution und den von ihr ausgehenden Gefahren. ${ }^{3}$ Die wenigen Politiker und Gelehrten in Portugal, die die Ereignisse von Saint Domingue überhaupt thematisierten, deuteten sie als Begleiterscheinung der Französischen Revolution. Deren „abscheuliche Prinzipien“ hätten auch zu den grausamen Erhebungen und bürgerkriegsartigen Zuständen in den französischen Kolonien geführt. ${ }^{4}$ Daher genüge es, das Eindringen von Ideen und Personen aus Frankreich zu verhindern, um Ereignisse wie die in Haiti abzuwehren. Doch tatsächlich hatten die Haitianer und Haitianerinnen ihre ganz eigenen Motive für die Revolution und gingen weitgehend unabhängig vom Geschehen in Frankreich vor. Für die königlichen Berater dürfte es jenseits des Vorstellungsvermögens gelegen haben, dass Schwarze die von Weißen errichtete gesellschaftliche Ordnung stürzen könnten. Michel-Rolph Trouillot spricht in diesem Sinn von einem „undenkbaren“ Ereignis. ${ }^{5}$ Die große Mehrheit der weißen Bevölkerung äußerste sich gar nicht zu Haiti - zum Teil dürfte dies einem mehr oder weniger bewussten Verdrängen geschuldet gewesen sein, zum Teil handelte es sich aber möglicherweise auch um eine Strategie, um Nachahmung zu vermeiden.

Wie schnell sich die Nachrichten über die Ereignisse auf Haiti dennoch unter den Schwarzen in Brasilien verbreiteten, macht ein Bericht aus dem Jahr 1805 deutlich. Nur wenige Monate, nachdem sich der ehemalige Sklave Jean-Jacques Dessalines zum Kaiser des unabhängigen Haiti erklärt hatte, sah sich ein Strafrichter (ouvidor do crime) in Rio veranlasst, einigen freien Schwarzen das Porträt von Dessalines von der Brust zu reißen. Am

3 Ein prominentes, ausführlich in der Historiographie behandeltes Beispiel ist Hegels Art über die beiden Ereignisse zu sprechen (beziehungsweise zu schweigen), vgl. Buck-Morss 2009.

4 So etwa der portugiesische Staatssekretär Martinho de Melo e Castro an den Vizekönig in Rio Graf von Resende in einem Brief vom 21.2.1792; Lara 1988, S. 35; vgl. dazu auch Marquese/Parron/Berbel 2016, S. 68.

5 "When reality does not coincide with deeply held beliefs, human beings tend to phrase interpretations that force reality within the scope of these beliefs. They devise formulas to repress the unthinkable and to bring it back within the realm of accepted discourse. [...] The Haitian Revolution thus entered history with the peculiar characteristic of being unthinkable even as it happened. [...] They [die Zeitgenossen] could read the news only with their ready-made categories, and these categories were incompatible with the idea of a slave revolution." Trouillot 1995/2015, S. $72 \mathrm{f}$. 
meisten erschütterte den Verfasser des Berichts, dass diese Männer den Milizen von Rio angehörten, wo sie die Artillerie bedienten und damit über direkten Zugriff auf Kampfmittel verfügten (Mott 1982, S. 57). Dennoch kam es weder zu einer Diskussion über die Schlussfolgerungen, die aus solchen Erfahrungen zu ziehen seien, noch gab es konkrete Handlungsanweisungen an die Polizei, die quellenmäßig belegt wären. Dies macht die Ambivalenz der Weißen gegenüber der Möglichkeit einer größeren schwarzen Revolte deutlich. Einerseits erkannten sie in den Schwarzen durchaus potenzielle Aufständische und fürchteten, dass das Vorbild Haiti ansteckend wirken könne. Andererseits trauten sie ihnen ein koordiniertes Vorgehen nicht zu; eine von Schwarzen durchgeführte Revolution passte nicht in ihr Weltbild.

\section{Schweigen über Differenz}

Das Ausklammern der versklavten und freien Schwarzen aus dem amtlichen Schriftgut ist ein Phänomen, das sich für die gesamte brasilianische Kolonialzeit (1500-1822) beobachten lässt. In abgeschwächter Form erstreckt es sich auch über die anschließende Zeit des Kaiserreichs (18221889) und tritt schließlich wieder intensiv in der Zeit der Republik (seit 1889) auf, zumindest bis zur Verabschiedung der Verfassung von 1988, in der erstmals die Multiethnizität der brasilianischen Nation anerkannt wurde. Das Schweigen über die schwarze Bevölkerung war allerdings nie absolut. Es gibt zahlreiche Kontexte beziehungsweise Quellengattungen, in denen Schwarze präsent sind, etwa im Zusammenhang mit dem transatlantischen Sklavenhandel oder in den Polizei- und Justizakten, die bei Festnahmen wegen Unruhestiftung oder im Rahmen von Freiheitsprozessen von Versklavten im 19. Jahrhundert entstanden. Doch im politischen Diskurs über das öffentliche Leben, die städtischen und staatlichen Institutionen und die Bevölkerung im Allgemeinen nahmen die Schwarzen lange eine sehr marginale Stellung ein. Wie ich in meiner Monographie über die Wasserversorgung von Rio de Janeiro zeige, wurden sie nicht als Teil des politischen Gemeinwesens wahrgenommen. ${ }^{6}$ Obwohl die Versorgung der einzelnen Haushalte mit Trinkwasser bis ins späte 19. Jahrhundert fast vollständig in den Händen von versklavten und freien Schwarzen lag, rich-

6 Jorun Poettering: Contested Waters, Shared Life. Water Supply and Society Formation in Rio de Janeiro, Sixteenth to Nineteenth Century (Arbeitstitel; erscheint voraussichtlich 2022). 
tete sich das Regierungshandeln bei der Schaffung der Infrastruktur, welche das Wasser für die Weiterverteilung zur Verfügung stellte, nur in wenigen Ausnahmefällen explizit an dieser Nutzergruppe aus. Besonders prägnant zeigt sich die Verleugnung der schwarzen Bevölkerung in der 1824 verabschiedeten ersten brasilianischen Verfassung. Sie erwähnt weder die Tatsache, dass sich die Bevölkerung Brasiliens aus unterschiedlichen ethnischen Gruppen zusammensetzte, noch, dass die Gesellschaftsordnung auf Sklaverei fußte. Sie definiert vielmehr, dass sie sich auf die brasilianische Nation beziehe, zu der gehöre, wer frei und in Brasilien geboren sei. Damit waren nicht nur alle Sklaven von der Nation und den in der Verfassung verbrieften Rechten (und Pflichten) ausgeschlossen, sondern auch alle Freigelassenen, die nicht in Brasilien geboren, sondern aus Afrika deportiert worden waren. Dennoch ist die Verfassung vergleichsweise inklusiv, denn sie legt fest, dass unter den so definierten Brasilianern ausschließlich die individuelle Leistung als Kriterium für die Besetzung der zivilen, militärischen und politischen Posten gelten solle (Fischer/Grinberg/Mattos 2018, S. 136). Das bedeutete die formale Gleichstellung aller (freien und in Brasilien geborenen männlichen) Brasilianer, unabhängig von ihrer Hautfarbe und Herkunft. Die Verfassung von 1824 unterscheidet nicht zwischen schwarzen und weißen Brasilianern; Differenz wird nicht benannt und damit die mit ihr einhergehende Diskriminierung auch nicht anerkannt.

Doch zurück zur Sicherheit: Anders als die Ausländer und die in Brasilien eintreffenden Portugiesen wurden die aus Afrika eingeführten Schwarzen ebenso wenig wie die in Brasilien geborenen versklavten (oder auch freien) Personen systematisch von den Behörden erfasst. Lediglich in ihrer Eigenschaft als zu verzollende Ware fanden sie bei der Einfuhr Eingang in die Register der Verwaltung, jedoch ohne dass eine individuelle Identifizierung stattgefunden hätte. Eine solche wäre auch gar nicht ohne weiteres möglich gewesen. Denn zum einen war die Zahl der in Rio eingeführten Sklaven mindestens zehnmal so groß wie die der freiwillig Ein- und Ausreisenden. ${ }^{7}$ Vor allem aber waren die versklavten Menschen nicht anhand der Kriterien kategorisierbar, mit denen die Weißen registriert wurden. So

7 Nach Gerstenberger wurden zwischen 1808 und 1822 insgesamt 28.234 ein- und ausreisende Ausländer und Portugiesen registriert; Gerstenberger 2013, S. 158f. Die Zahl der in Südwestbrasilien (d.h. im Wesentlichen in Rio de Janeiro) im selben Zeitraum eingeführten Sklaven betrug laut der Slave Trade Database 332.129; Slave Voyages 2020. In den folgenden Dekaden stieg die Zahl der eingeführten Sklaven deutlich an, während dies für die frei Eingereisten nicht der Fall gewesen sein dürfte. 
trugen die aus Afrika verschleppten Personen keine eindeutigen amtlich verifizierbaren Namen. Vielmehr erhielten sie nach ihrer Versklavung einen christlichen Taufnamen, aber keinen Nachnamen (Palma/Truzzi 2018, S. 314). Statt durch den Geburtsort bestimmte sich ihre Herkunft anhand einer „Nation“, die aus der Herkunftsregion, dem Ort des Sklavenmarktes, über den sie gekommen waren, dem afrikanischen Ausfuhrhafen, der Muttersprache oder anderen ethnischen Markern in keineswegs eindeutiger Weise abgeleitet wurde (Gomes 2011). Die bei den freiwillig Einreisenden abgefragte Kontaktperson hätte vielleicht der Sklavenhändler abgeben können, doch nach dem erstmaligen oder auch wiederholten Verkauf dürfte sich die Spur der Versklavten bald verloren haben. Anders als die freie Bevölkerung waren die Versklavten für die Behörden nicht „lesbar“, um mit James C. Scott (1998) zu sprechen. Im Alltag wurde dies durch die pauschalisierte „Lesbarkeit“ ihrer Hauptfarbe kompensiert, die jedoch in vielfacher Weise Unschärfe erzeugte und insbesondere nicht erlaubte, zwischen freien und versklavten Schwarzen zu unterscheiden.

Auch wenn die Schwarzen aus dem amtlichen Schriftgut weitgehend ausgeklammert waren und insbesondere die Sicherheitsmaßnahme der Registrierung nicht auf sie angewendet wurde und auch gar nicht ohne weiteres hätte angewendet werden können, stellten sie für die Weißen ein Sicherheitsrisiko dar, und zwar ein erhebliches. Dies galt nicht erst seit der Haitianischen Revolution, sondern im Grunde seit dem Beginn der weißen Präsenz in Brasilien, mit der auch die Identifizierung zunächst der Indigenen und dann der Afrikaner als "Schwarze“ (negros da terra bzw. negros da Guiné) einherging, also seit dem frühen 16. Jahrhundert. Mit dem äußerst zähen Kampf der kolonialen Streitkräfte gegen den Quilombo dos Palmares, einer Ansammlung von Siedlungen mit Tausenden entlaufener ehemals versklavter, aber auch freier Schwarzer im Nordosten Brasiliens, war diese Gefahr Ende des 17. Jahrhunderts besonders akut aufgeleuchtet (Vainfas 1996, S. 62f.). Aber auch sonst brachen immer wieder Sklavenrevolten aus, dazu kamen vielfältige individuelle Grenzüberschreitungen wie Gewaltakte gegen Besitzer und Besitzerinnen, mutwillige Zerstörungen, Diebstahl und immer wieder Flucht. In Rio de Janeiro schien die Bedrohung besonders intensiv, denn dort stellten die versklavten Menschen um die Mitte des 19. Jahrhunderts rund $40 \%$ der Gesamtbevölkerung (Soares 2007, S. 363-370), und anders als auf den Plantagen standen sie nicht unter der ständigen Beobachtung von Aufsehern, sondern konnten sich relativ frei in der Stadt bewegen.

Wie ging die weiße Gesellschaft gegen diese Gefahren vor? Selbst wenn Sicherheitsüberlegungen im öffentlichen Diskurs nur einen marginalen Platz einnahmen, ist davon auszugehen, dass die Sklavenbesitzer und -be- 
sitzerinnen die Gefahren wahrnahmen. Lange Zeit traten sie ihnen vor allem auf privater Ebene entgegen: Sklaven waren persönlicher Besitz und jeder war für seine Sklaven selbst verantwortlich. Sklavenbesitzer konnten und mussten sie nach eigenem Gutdünken durch Überwachung und Züchtigung, aber auch - was meist erfolgreicher gewesen sein dürfte - durch gute Behandlung, Belohnungen und das Versprechen der Freilassung in Schach halten. Bekam ein Sklavenhalter einen Sklaven oder eine Sklavin dauerhaft nicht unter Kontrolle, verkaufte er ihn nach außerhalb. Lief eine versklavte Person weg, konnten private Sklavenjäger sie wieder einfangen und dem vormaligen Besitzer gegen ein entsprechendes Lösegeld zurückgeben. Für freie Schwarze bedeutete das, dass sie ständig in Gefahr waren, versklavt zu werden, wenn sie ihre Freilassung oder freie Geburt nicht nachweisen konnten. Freilassungen wurden zwar in der Regel notariell dokumentiert, dennoch vermieden es staatliche Verwaltung und städtische Obrigkeit, sich in die Beziehung zwischen Herr beziehungsweise Herrin und Sklave beziehungsweise Sklavin mehr als unbedingt nötig einzumischen. Eine Gesetzgebung zum Schutz der Versklavten, die etwa dem französischen Code Noir entsprochen hätte, gab es in Brasilien nicht. Das Leben der Schwarzen spielte sich lange Zeit unterhalb des staatlichen Radars ab; sie waren ebenso wenig Gegenstand staatlichen Handelns wie staatlichen Sprechens.

\section{Schwarze als Ziel staatlicher Repression und als Einfordernde von Rechtssicherheit}

Tatsächlich war es eben jener 1808 eingesetzte Polizeiintendant, der die Schwarzen auf städtischer Ebene erstmals massiv mit der Staatsgewalt in Berührung brachte - und zwar, weil sie es waren, die seiner Ansicht nach die "öffentliche Ruhe“ störten, zu deren Wahrung er eingesetzt worden war. Auswertungen der Polizeiakten aus den Jahren 1810 bis 1821 zeigen, dass die Polizei in diesem Zeitraum fast ausschließlich Schwarze verhaftete. Rund $80 \%$ der Häfllinge waren Sklaven, rund 19\% Freigelassene, und nur bei $1 \%$ der Verhafteten handelte es sich um Personen, die nie versklavt gewesen waren (und bei denen die Hautfarbe nicht bekannt ist). ${ }^{8}$ Der britische Geschäftsmann John Luccock beobachtete dementsprechend während seines Aufenthaltes in Rio von 1808 bis 1818, dass sich die weiße Bevölkerung zunehmend in ihrer Selbstwahrnehmung bestätigt fühle,

8 Algranti 1988a, S. 157-201, 209-211, zit. n. Holloway 1993, S. 38 f. 
über dem Gesetz zu stehen, und dass dies selbst auf „Mulatten“ zutreffe, je mehr sich ihre Hautfarbe dem „Trüben“ (dingy) oder „Hellen“ (fair) annähere. ${ }^{9}$ Die meisten Verhaftungen schwarzer Personen erfolgten wegen ordnungswidrigen Verhaltens, Gelegenheitsdiebstählen, des Tragens von Waffen, Trunkenheit und ähnlicher Vergehen (Algranti 1988b, S. 31). Der de facto-Einbeziehung der Versklavten in das Staatswesen folgte mit dem 1830 veröffentlichten ersten Strafgesetzbuch auch ihre de jure-Einbeziehung. Denn anders als die Verfassung erklärte es Sklaven zu einer eigenen Statusgruppe, deren Angehörige als eigenständige juristische Personen die volle Verantwortung für ihre Taten trugen und deutlich schärferen Strafen unterworfen waren als die übrige Bevölkerung (Fischer/Grinberg/Mattos 2018, S. 137, 139).

An dem fast ausschließlich auf die schwarze Bevölkerung konzentrierten Vorgehen der Polizei dürfte sich bis zum Ende des Kaiserreichs wenig geändert haben (Abb. 1). Insbesondere die Teilnahme an dem noch heute beliebten Kampf-Tanz Capoeira, bei dem je zwei capoeiristas ihre Fertigkeiten innerhalb eines Kreises von weiteren Teilnehmern und Musikern vorführen, war ein häufiger Haftgrund, obwohl er erst 1890 offiziell verboten wurde (Holloway 1993, S. 8; Soares 2004). Schwarze waren also weit überproportional von der polizeilichen Repression betroffen, kamen dadurch aber auch immer stärker mit staatlichen Institutionen in Kontakt, die bis dahin für die Untertanen des portugiesischen Königs beziehungsweise die weißen Bürger des brasilianischen Kaiserreichs reserviert gewesen waren. Oder, anders ausgedrückt: Über das Sicherheitsinstrument einer repressiven Polizei fanden die Schwarzen Eingang in das öffentlich-staatliche Handeln und in der Folge auch in den entsprechenden Diskurs.

Parallel zu dieser Entwicklung richtete sich die schwarze Bevölkerung selbst immer häufiger an staatliche Institutionen, um Rechte oder Freiheit einzuklagen. Versklavte Personen konnten hierbei, wie erwähnt, nicht auf eine positive Gesetzesgrundlage zurückgreifen, wohl aber auf ein allmählich etabliertes Gewohnheitsrecht. Im 18. Jahrhundert waren entsprechende Gerichtsprozesse oft auf die Initiative von Weißen zurückgegangen, die die Freilassung ehemaliger Sklaven wegen „Undankbarkeit“ rückgängig

9 "The laws were so imperfect, or so imperfectly executed, that white people seemed to have been gradually confirmed in the persuasion, that they were above their influence. Few of that class had, I believe, been recently apprehended for crimes, except those committed against the State; and Mulattoes enjoyed like exemptions, in proportion as their colour approached the dingy or fair." Luccock 1820, S. 137. 


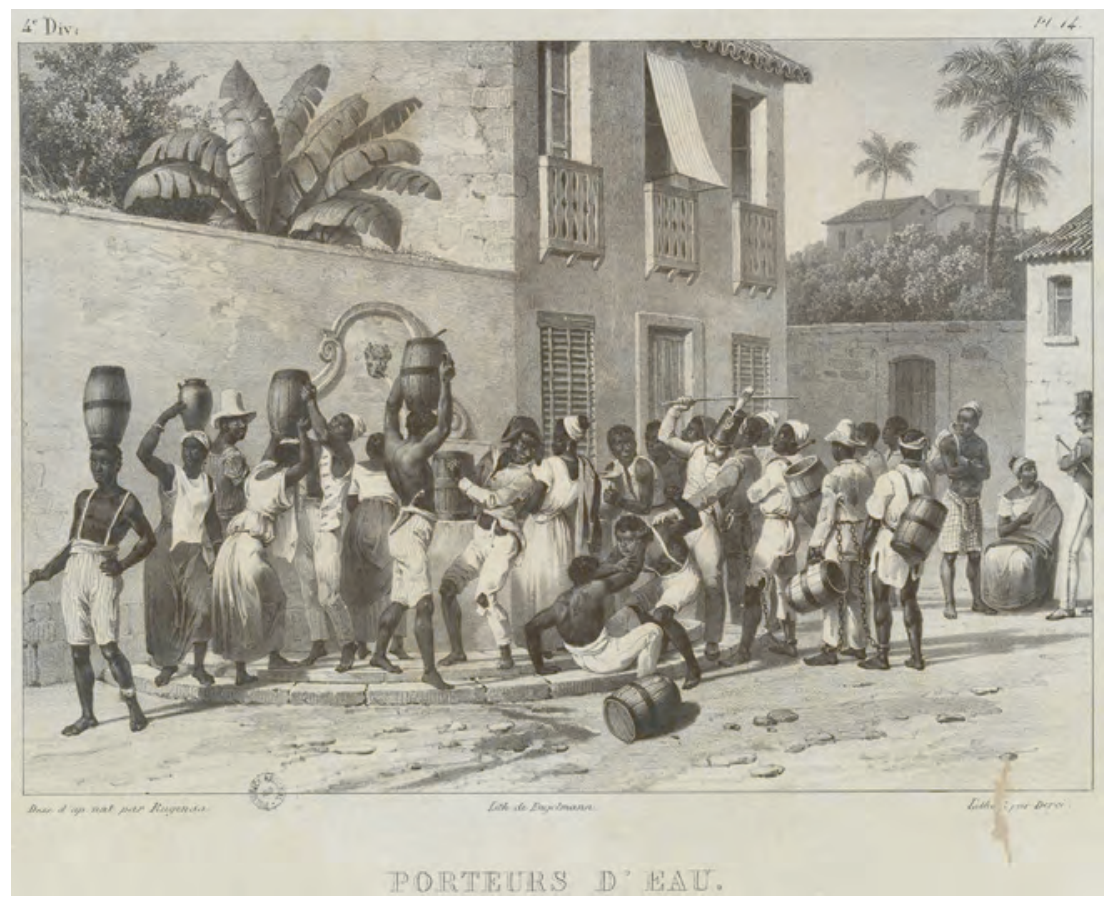

Abb. 1: Eingreifen eines Polizeioffiziers in einen Streit schwarzer Wasserträger an einem Brunnen in Rio de Janeiro, dargestellt auf einer Lithographie von Laurent Deroy nach einer Vorlage von Johann Moritz Rugendas, "Porteurs d'eau", aus: Johann Moritz Rugendas: Voyage pittoresque dans le Brésil. Paris: Engelmann \& Cie. 1835, S. [Graphik] 93 (Biblioteca Nacional, Rio de Janeiro, Iconografia; http://acervo.bndigital.bn.br/sophia/index.asp?codigo_sophia=6213)

machen wollten (Pinheiro 2016). ${ }^{10}$ Im 19. Jahrhundert wandten sich die Schwarzen zunehmend selbst an die Autoritäten, um ihre Rechte durchzusetzen, insbesondere bei illegalen Versklavungen, oder wenn Sklavenbesitzer oder -besitzerinnen Abmachungen über Freikäufe oder bedingte Freilassungen brachen (Chalhoub 1990). Nicht selten bekamen die Schwarzen Recht. ${ }^{11}$ Dadurch wuchs die Rechtsgültigkeit entsprechender Abmachungen, und die Versklavten wurden allmählich zu Rechtssubjekten, die nicht

$10 \mathrm{Zu}$ Fällen aus dem 18. Jahrhundert, bei denen die Initiative von den Schwarzen ausging, vgl. etwa Pinheiro 2018.

11 In fast der Hälfte der Fälle, die vor das Appellationsgericht von Rio de Janeiro kamen, erhielten die Schwarzen die Freiheit; Grinberg 2008/2010, S. 13. 
nur zur Rechenschaft gezogen werden, sondern auch Sicherheit einfordern konnten. 1871 wurde schließlich das "Gesetz des freien Bauches“ (Lei do ventre livre) verabschiedet, demzufolge alle von diesem Zeitpunkt an von versklavten Frauen geborenen Kinder frei waren. Außerdem bestätigte das Gesetz einige der Gewohnheitsrechte, darunter das Recht, Geld sparen und sich freikaufen zu dürfen. Auch setzte es das bis dahin gültige Recht ehemaliger Besitzer außer Kraft, eine Freilassung wegen „Undankbarkeit“ rückgängig machen zu können. Das Gesetz von 1871 gestand den versklavten Personen das Recht auf Rechte zu und besaß daher auch eine große symbolische Bedeutung (Fischer/Grinberg/Mattos 2018, S. 137; Chalhoub 2016, S. 202).

\section{Markierung von Differenz und Ende der Sklaverei}

Diese Entwicklungen schlugen sich schließlich auch in der Sichtbarkeit der Versklavten im allgemeinen staatlichen Verwaltungsschriftum nieder. 1872 fand der erste nationale Zensus in Brasilien statt. Er orientierte sich im Wesentlichen an den wenig zuvor in Europa entwickelten wissenschaftlichen Kriterien für entsprechende Bevölkerungserhebungen, war jedoch um einige spezifisch brasilianische Elemente ergänzt worden (Recenseamento [1876]; Loveman 2009, S. 435). Insbesondere führte er Angaben zur „Kondition“ (condição) der Personen an, also ob sie frei oder versklavt waren, zur Hautfarbe beziehungsweise ethnischen Zugehörigkeit, die weiß (branco), braun (pardo, mestiço), schwarz (preto, negro, africano) oder indigen (caboclo, indigena) sein konnte, und schließlich zum Schulbesuch. Die „Kondition“ erhielt allerdings erst im Zuge der statistischen Auswertung Gewicht, denn in den zugrundeliegenden Fragebögen (Abb. 2), die jeweils pro Haushalt ausgefüllt werden mussten, sollten außer den Mitgliedern der jeweiligen Kernfamilie zwar auch Bedienstete (criados), Sklaven (escravos), Untermieter (agregados) oder Gäste (bóspedes) aufgelistet werden, doch was sie genau waren, musste nicht spezifiziert werden. In der Spalte neben den Namen sollte für jede Person die Hautfarbe angegeben werden, doch für die „Kondition“ war keine Spalte vorgesehen. Die Angabe, ob jemand "Sklave“ war, wurde ausschließlich in der für „Bemerkungen“ vorgesehenen letzten Spalte gemacht (in der laut Formular etwa der eigentliche Wohnort der Gäste, etwaige Behinderungen oder ähnliches angegeben werden sollten) (Camargo 2018, S. 420f.).

In der vier Jahre später veröffentlichten Auswertung hatte die Unterscheidung zwischen „Freien“ und „Sklaven“ dagegen oberste Priorität; in der Generalübersicht standen sich die freie und die versklavte Bevölkerung 


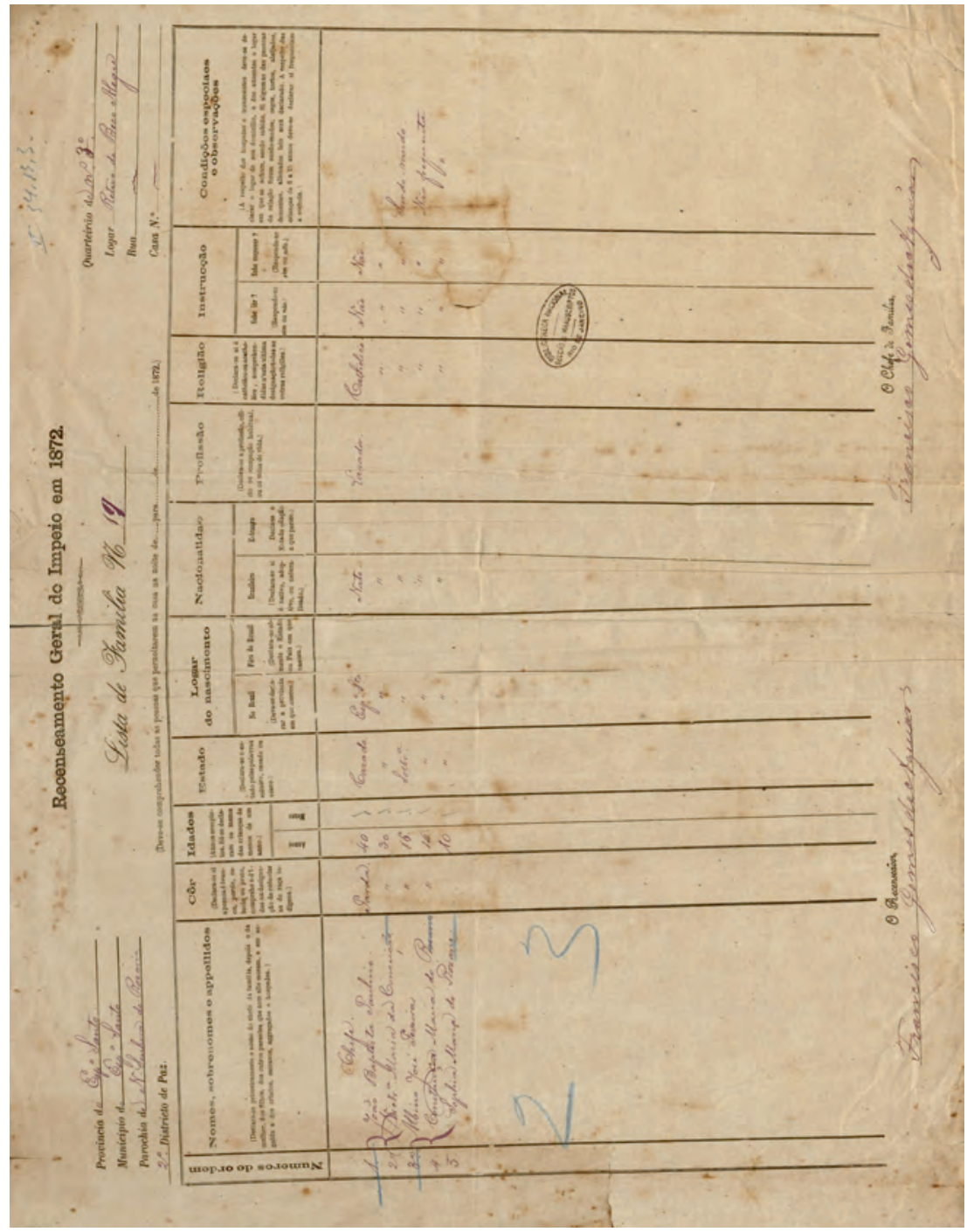

Abb. 2: Beispiel für einen Fragebogen von 1872, in diesem Fall obne Sklaven [Resultado do recenseamento geral do império de 1872, no municipio do Espírito Santo, paróquia de Nossa Senhora do Rosário, no quarteirão no 3, Retiro do Beco Alegre] (Biblioteca Nacional, Rio de Janeiro, II-34,13,003, Manuscritos; http://acervo.bndigital.bn.br/sophia/index.as p?codigo_sophia=82801). 
in zwei vollständig voneinander getrennten Tabellen gegenüber, die sich bezüglich der Unterkategorien leicht unterschieden. ${ }^{12}$ Anders als bei den Einreiseregistern der ersten Hälfte des Jahrhunderts waren nun also beide Teile der Bevölkerung erfasst und klar differenziert. Sicherheitsüberlegungen waren jedoch nicht der Grund für diese Darstellungsart. Vielmehr war ein Jahr bevor der Zensus durchgeführt wurde, das erwähnte „Gesetz des freien Bauches" verabschiedet worden, welches das Ende der Sklaverei absehbar machte. Zwischen dem Entwurf und der Auswertung des Zensus hatte sich die Regierung dazu entschlossen, eine Politik zu entwerfen (bzw. zu legitimieren), welche die zu diesem Zeitpunkt noch versklavten Menschen langfristig in den freien Arbeitsmarkt integrieren würde, um die wirtschaftliche Entwicklung des Landes auch ohne Sklaverei vorantreiben zu können. Grundlage für die Planung dieser Politik sollte der Zensus sein (Loveman 2009, S. 440; Camargo 2018, S. 420, 426). ${ }^{13}$

Der Zensus von 1872 stand zwar nicht mit Sicherheitsüberlegungen der Behörden in Zusammenhang, half jedoch bei der Einrichtung eines Melderegisters für Sklaven (matrícula geral dos escravos), das die Rechtssicherheit der freien Schwarzen erheblich vergrößerte. ${ }^{14}$ Denn illegale Versklavungen wurden damit besser feststellbar und Freiheit leichter einklagbar. Ein solches Register hätte sich jedoch auch negativ auf die Sicherheit der Schwarzen auswirken können. Tatsächlich hatte es bereits 1851 Bemühungen gegeben, ein allgemeines Melderegister auf der Grundlage eines bundesweiten Zensus einzuführen. Diese Bemühungen hatten jedoch zu erheblichen Unruhen geführt, die wohl im Wesentlichen auf die Befürchtung freier Schwarzer zurückgingen, dass mit Hilfe dieses Registers ihre massenhafte (Wieder-)Versklavung durchgeführt werden sollte. Denn ein Jahr zuvor hatte der transatlantische Sklavenhandel sein abruptes und definitives Ende gefunden, nachdem die Importe zuletzt bei durchschnittlich 50.000 Personen pro Jahr gelegen hatten. ${ }^{15}$ Eine deutliche Verknappung der Arbeitskräfte war also abzusehen und eine Versklavung von freien Schwarzen in Brasilien denkbar. Allerdings täuschten sich die Unruhestifter: die Regierung verfolgte keine solchen Absichten (Chalhoub 2018).

12 So konnten Freie beispielsweise „katholisch“ oder „akatholisch“ sein, während Sklaven grundsätzlich „katholisch“"waren; Recenseamento [1876].

13 Ein weiterer Anlass war das Ende des Paraguay-Krieges (1864-1870); der Umfang der verbleibenden rekrutierbaren Bevölkerung sollte festgestellt werden.

14 Die Aufstellung eines solchen Registers war durch das Gesetz des freien Bauches im Vorjahr angeordnet worden. Espíndola 2015; Camargo 2018, S. 426.

15 Es handelt sich um den Durchschnitt der lebend in Brasilien eingetroffenen Afrikaner und Afrikanerinnen in den Jahren 1845 bis 1859; Slave Voyages (2020). 
Auch wenn der Zensus von 1872 die Versklavten sichtbar machte und zum Schutz der freien Schwarzen beitrug, kann auch er kritisch gesehen werden. Denn wie immer bei solchen Kategorisierungen vereinfachte die Aufteilung der Bevölkerung in freie und unfreie Menschen eine deutlich komplexere Situation. Die Freiheit von Schwarzen war nach wie vor prekär und konnte unter Umständen rückgängig gemacht werden. Zudem gab es viele Menschen, die sich in einer Grauzone zwischen Freiheit und Unfreiheit befanden, etwa wenn sie sich bereits zu einem bestimmten Anteil freigekauft oder bereits einige der vereinbarten Bedingungen für ihre Freilassung erfüllt hatten (Cantisano/Paes 2018; Chalhoub 2011). Dem Historiker Sidney Chalhoub zufolge ist die scharfe Unterscheidung zwischen Sklaven und Freien ein ideologisches Konstrukt, das es erlaubt, die Abschaffung der Sklaverei als Wendepunkt in der Geschichte Brasiliens zu feiern. Die Unterscheidung verhindere jedoch die angemessene Wahrnehmung der Bedingungen und Abhängigkeiten, unter denen die versklavten Schwarzen leben mussten, ebenso wie derjenigen, unter denen nominell freie Arbeiter in Brasilien bis heute leben müssen (Chalhoub 2016, S. 184, 213f.).

\section{Das Schweigen kehrt zurück}

Die brasilianische Sklaverei fand 1888 ihr offizielles Ende. In den nachfolgenden Zensus erübrigte sich daher die Unterscheidung in Sklaven und Freie; doch auch die Hautfarbe beziehungsweise die ethnische Zugehörigkeit wurde im Zensus von 1900 nicht mehr angegeben und gehörte auch im folgenden Zensus von 1920 nicht zu den vorrangigen Kriterien (Loveman 2009, S. 454, 460). Dies suggeriert Gleichstellung, doch tatsächlich war die Weglassung rassistisch motiviert. In den letzten beiden Dekaden des 19. Jahrhunderts hatten auch in Brasilien Rassentheorien nach europäisch-nordamerikanischem Vorbild Einzug gehalten (Schwarcz 1993). Die brasilianische Nation sollte möglichst „hell“" sein; durch das Schweigen der Zensus über die ethnische Vielfalt erschienen die Brasilianer „normaler“ und damit „weißer“. Zudem sollte die schwarze Bevölkerung nun nicht mehr (wie noch bei der Auswertung des Zensus von 1872) in den Arbeitsmarkt integriert werden. Stattdessen verfolgte die Regierung eine Politik der „Aufweißung“ (branqueamento), welche die ehemalige Arbeitskraft versklavter Personen durch die massenhafte Anwerbung weißer Einwanderer aus Europa zu ersetzen suchte, die nicht nur als fleißiger und besser ausgebildet galten, sondern sich aufgrund ihrer angeblich überlegenen Gene auch demographisch gegenüber der schwarzen Bevölkerung durchsetzen 
würden. Im Zensus von 1890 wurde daher an Stelle der ethnischen Zugehörigkeit der Geburtsort der Eltern abgefragt, denn nur daraus ließ sich Information über die europäische Immigration ableiten (Loveman 2009, S. 449). Die schwarzen Menschen in Brasilien waren dagegen aus Sicht der Regierung überflüssig geworden.

Gerade wegen des immer stärker werdenden Rassismus verschwand also die Differenzierung nach Hautfarbe Ende des 19. Jahrhunderts fast vollständig aus der amtlichen Dokumentation. Im Rahmen des Abolitionismus hatte sich eine ursprünglich durchaus emanzipatorisch intendierte „Ethik des Schweigens“ etabliert, die freie Schwarze nicht aufgrund ihrer Hautfarbe stigmatisieren wollte. Sie wurde bald von der Regierung übernommen, doch diese löste das damit verbundene Versprechen formaler Gleichheit nicht ein (Fischer/Grinberg/Mattos 2018, S. 142f.). Denn auch wenn die Hautfarbe aus allen amtlichen Vorgängen entfernt wurde, war die schwarze Bevölkerung sowohl im Alltag als auch in der Rechtspraxis erheblichen Diskriminierungen ausgesetzt; insbesondere erlitt und erleidet sie bis heute weit mehr Gewalt durch die Polizei als die weiße Bevölkerung. Gerade weil es dafür keine gültige Sprache gab, nahmen viele Betroffene, aber auch viele Soziologen den rassistischen Grundton der gesellschaftlichen Wirklichkeit lange nicht wahr. Da es offiziell keine „Rassen“ gab, wurden auch keine Daten wegen rassistischer Diskriminierung gesammelt, keine Beschwerdestellen eingerichtet und entsprechende Vorkommnisse nicht geahndet. Vielmehr gab es seit den 1930er Jahren gerade unter den Geistes- und Sozialwissenschaftlern sogar einen gewissen Stolz, dass in Brasilien alle Menschen gleichgestellt seien, und das Land sich damit positiv von den USA und deren Jim Crow-Gesetzen abhebe. Es kam der Mythos der "Rassendemokratie“ (democracia racial) auf, die angeblich in Brasilien herrsche und sich in der Gleichstellung aller „Rassen“ sowie einer besonderen „Herzlichkeit“ (cordialidade) zwischen ihren Mitgliedern äußere. Schwarze Intellektuelle, die sich gegen entsprechende Interpretationen wehrten, kämpften lange auf verlorenem Posten (ebd., S. 142-145). Erst die Verfassung von 1988 ebnete, wie erwähnt, den Weg für die Anerkennung und den Schutz aller ethnischen Gruppen und für die juristische Aufarbeitung rassistisch bedingter Ungerechtigkeiten und Ungleichheiten. Dennoch sprechen (schwarze und weiße) Intellektuelle und Aktivisten heute, nicht zuletzt anlässlich der COVID-19-Pandemie, von einem Geno- 
zid, der an der schwarzen Bevölkerung Brasiliens verübt werde - nach wie vor ohne ernsthaft Gehör zu finden. ${ }^{16}$

Die durch wissenschaftliche oder staatliche Institutionen durchgeführte Einteilung der Bevölkerung in Kategorien kann soziale Realität immer nur unzureichend beschreiben und konstituiert diese zum Teil überhaupt erst. Dennoch können solche Kategorien ein notwendiges Werkzeug sein, um gegen faktisch bestehende Ungleichheiten und Ungerechtigkeiten anzugehen. Differenz zu benennen kann inklusiv wirken und den Weg bereiten, um Sicherheit für subalterne Menschen zu erzeugen (insbesondere gegenüber dem staatlichen Sicherheitsapparat); und idealerweise löst diese Sicherheit die Differenz dann auf. Oder, anders ausgedrückt: Oft existiert eine faktische Sicherheitssituation neben dem offiziellen Sicherheitsdiskurs, der sich nur auf ausgewählte gesellschaftliche Sektoren oder den Staat als solchen bezieht. Wer faktische Sicherheit für alle möchte, ist darauf angewiesen, über Differenz (etwa im Sinne der Rechtstellung oder rassistisch bedingter Erfahrungen) zu sprechen und den Subalternen zuzuhören. Diese Aufgabe übernahmen in Brasilien insbesondere die Anwälte, die die Versklavten in ihren Freiheitsprozessen vertraten. Gemeinsam mit den Schwarzen spielten sie eine fundamentale Rolle auf dem Weg zu deren individueller Freiheit, zur Abschaffung der rechtlich legitimierten Sklaverei und zur Etablierung des brasilianischen Rechtsstaates.

16 „Völkermord in Brasilien“. Offener Brief von Frei Betto, 21.7.2020. blog.misereor.de/2020/07/21/voelkermord-in-brasilien-offener-brief-von-frei-betto, 25.08.2020; Beitrag von Adilson José Moreira in: PUB Boston Especial - Debate Racismo no Brasil, 26.6.2020. www.pubboston.org/new-events/2020/6/26/pub-boston-espec ial-debate-racismo-no-brasil, 01.07. 2020. Der Vorwurf erhielt mit der COVID-19Krise erhöhte Dringlichkeit, ist jedoch keineswegs neu, vgl. etwa Nascimento $1978 / 2016$. 


\section{Literatur}

Algranti, Leila Mezan (1988a): O feitor ausente. Estudos sobre a escravidão urbana no Rio de Janeiro, 1808-1822. Petrópolis: Vozes.

Algranti, Leila Mezan (1988b): Slave Crimes. The Use of Police Power to Control the Slave Population of Rio de Janeiro. In: Luso-Brazilian Review 25, H. 1, S. 27-48.

Auler, Guilherme (Hrsg.) (1960): Registro de estrangeiros, 1808-1822. Rio de Janeiro: Arquivo Nacional.

Bertrand, Sarah (2018): Can the Subaltern Securitize? Postcolonial Perspectives on Securitization Theory and its Critics. In: European Journal of International Security 3, H. 3, S. 281-299.

Buck-Morss, Susan (2009): Hegel, Haiti and Universal History. Pittsburgh (PA): University of Pittsburgh Press.

Camargo, Alexandre de Paiva Rio (2018): O censo de 1872 e a utopia estatística do Brasil Imperial. In: História Unisinos 22, H. 3, S. 414-428.

Cantisano, Pedro Jimenez/Paes, Mariana Armond Dias (2018): Legal Reasoning in a Slave Society (Brazil, 1860-88). In: Law and History Review 36, H. 3, S. 471510.

Chalhoub, Sidney (1990): Visões da liberdade. Uma história das últimas décadas da escravidão na corte. São Paulo: Companhia das Letras.

Chalhoub, Sidney (2011): The Precariousness of Freedom in a Slave Society (Brazil in the Nineteenth Century). In: International Review of Social History 56, H. 3, S. 405-439.

Chalhoub, Sidney (2016): The Politics of Ambiguity. Conditional Manumission, Labor Contracts, and Slave Emancipation in Brazil (1850s-1888). In: Eckert, Andreas (Hrsg.): Global Histories of Work. Berlin: De Gruyter Oldenbourg, S. 183214.

Chalhoub, Sidney (2018): The Great Fear of 1852. Riots against Enslavement in the Brazilian Empire. In: Bosma, Ulbe/Hofmeester, Karin (Hrsg.): The Lifework of a Labor Historian. Essays in Honor of Marcel van der Linden. O.O.: Brill, S. 115135.

Collecção (1891): Collecção das leis do Brazil, Teil 1 (Cartas de lei, alvarás, decretos e cartas régias 1808). Rio de Janeiro: Imprensa Nacional.

Domingues, Ângela (2013): Oficiais, cavalheiros e concorrentes. O "Brasil" nas viagens de circum-navegação do século das Luzes. In: Revista das Índias 73, H. 258, S. 365-398.

Espíndola, Ariana Moreira (2015): Papéis da escravidão. A matrícula especial de escravos (lei de 1871). In: Anais do $7^{\circ}$ encontro escravidão e liberdade no Brasil meridional. Curitiba: Universidade Federal do Paraná.

Fischer, Brodwyn/Grinberg, Keila/Mattos, Hebe (2018): Law, Silence, and Racialized Inequalities in the History of Afro-Brazil. In: Fuente, Alejandro de la/ Andrews, George Reid (Hrsg.): Afro-Latin American Studies. An Introduction. Cambridge: Cambridge University Press, S. 130-176. 
Gerstenberger, Debora (2013): Gouvernementalität im Zeichen der globalen Krise. Der Transfer des portugiesischen Königshofes nach Brasilien. Köln: Böhlau.

Gomes, Flávio dos Santos (2011): “Atlantic Nations” and the Origins of Africans in Late-Colonial Rio de Janeiro. New Evidence. In: Colonial Latin American Review 20, H. 2, S. 213-231.

Grinberg, Keila (2008/2010): Liberata. A lei da ambiguidade - as ações de liberdade da corte de apelação do Rio de Janeiro no século XIX. Rio de Janeiro: Centro Edelstein de Pesquisa Social.

Hansen, Lene (2000): The Little Mermaid's Silent Security Dilemma and the Absence of Gender in the Copenhagen School. In: Millennium. Journal of International Studies 29, H. 2, S. 285-306.

Härter, Karl (2013): Security and Cross-Border Political Crime. The Formation of Transnational Security Regimes in 18th and 19th Century Europe. In: Historical Social Research 38, H. 1, S. 96-106.

Holloway, Thomas H. (1993): Policing Rio de Janeiro. Repression and Resistance in a 19th-Century City. Stanford (CA): Stanford University Press.

Lara, Silvia Hunold (1988): Campos da violência. Escravos e senhores na capitania do Rio de Janeiro 1750-1808. Rio de Janeiro: Paz e Terra.

Loveman, Mara (2009): The Race to Progress. Census Taking and Nation Making in Brazil (1870 - 1920). In: Hispanic American Historical Review 89, H. 3, S. 435-470.

Luccock, John (1820): Notes on Rio de Janeiro and the Southern Parts of Brazil. Taken during a Residence of Ten Years in that Country, from 1808 to 1818. London: Samuel Leigh.

Marquese, Rafael de Bivar/Parron, Tâmis/Berbel, Márcia Regina (2016): Slavery and Politics. Brazil and Cuba, 1790-1850. Albuquerque: University of New Mexico Press.

Mott, Luiz R. B. (1982): A revolução dos negros do Haiti e do Brasil. In: História. Questões \& Debates 3, H. 4, S. 55-63.

Nascimento, Abdias do (1978/2016): O genocídio do negro brasileiro. Processo de um racismo mascarado, eingeleitet v. Florestan Fernandes u. Wole Soyinka, Nachwort v. Elisa Larkin Nascimento. São Paulo: Perspectiva S.A.

Palma, Rogerio da/Truzzi, Oswaldo (2018): Renomear para recomeçar. Lógicas onomásticas no pós-abolição. In: DADOS. Revista de Ciências Sociais 61, H. 2 , S. 311-340.

Recenseamento ([1876]). Recenseamento do Brazil em 1872. 12 Bde. Rio de Janeiro: G. Leuzinger.

Santos, Luiz Gonçalves dos (1943): Memórias para servir à História do Reino do Brasil, hg. v. Noronha Santos, Bd. 1. Rio de Janeiro: Zélio Valverde.

Slave Voyages (2020): Slave Voyages. The Trans-Atlantic Slave Trade Database. slavevoyages.org/assessment/estimates, 20.08.2020.

Spivak, Gayatri Chakravorty (1988/2016): Can the Subaltern Speak? In: Dies. (Hrsg.): Can the Subaltern Speak? Postkolonialität und subalterne Artikulation. Wien: Turia + Kant, S. 17-118. 
Pinheiro, Fernanda Domingues (2016): Libertos ingratos. Práticas de redução ao cativeiro na América portuguesa (século XIII). In: Xavier, Ângela Barreto/Silva, Ana Cristina Nogueira da (Hrsg.): O governo dos outros. Poder e diferença no império português. Lissabon: ICS, S. 365-386.

Pinheiro, Fernanda Domingues (2018): The Threat of Reenslavement. Legal Battles to Maintain Freedom in Seventeenth-Century Mariana, Brazil. In: Revista Brasileira de História 38, H. 79, S. 1-22.

Schwarcz, Lilia Moritz (1993): O espetáculo das raças. Cientistas, instituições e questão racial no Brasil 1870-1930. São Paulo: Companhia das Letras.

Scott, James C. (1998): Seeing like a State. How Certain Schemes to Improve the Human Condition Have Failed. New Haven: Yale University Press.

Soares, Carlos Eugênio Líbano (2004): A capoeira escrava e outras tradições rebeldes no Rio de Janeiro (1808-1850). 2. überarb. u. erw. Aufl., Campinas (SP): UNICAMP.

Soares, Luiz Carlos (2007): O “povo de Cam” na capital do Brasil. A escravidão urbana no Rio de Janeiro do século XIX. Rio de Janeiro: 7Letras.

Torpey, John C. (2018): The Invention of the Passport. Surveillance, Citizenship and the State. 2. Aufl., Cambridge: Cambridge University Press.

Trouillot, Michel-Rolph (1995/2015): Silencing the Past. Power and the Production of History. Boston (MA): Beacon Press.

Vainfas, Ronaldo (1996): Deus contra Palmares. Representações senhoriais e idéias jesuíticas. In: Reis, João José/Gomes, Flávio dos Santos (Hrsg.): Liberdade por um fio. História dos quilombos no Brasil. São Paulo: Companhia das Letras, S. $60-80$.

Viana, Larissa (2007): O idioma da mestiçagem. As irmandades de pardos na América Portuguesa. Campinas (SP): UNICAMP. 